\title{
Cuatro décadas de políticas para integrar las tecnologías digitales en el aula en Cataluña: acciones, logros y fracasos
}

\author{
Begoña Gros \\ bgros@ub.edu \\ Universitat de Barcelona \\ Joan-Anton Sánchez Valero \\ joananton.sanchez@ub.edu \\ Universitat de Barcelona \\ Iolanda García \\ igarciago@uoc.edu \\ Universitat Oberta de Catalunya \\ Cristina Alonso Cano \\ cristina.alonso@ub.edu \\ Universitat de Barcelona
}

\begin{abstract}
Resumen
En la mayoría de los países europeos, la implementación de las tecnologías digitales en educación ha estado generalmente impulsada a través de políticas gubernamentales. En este artículo nos centraremos en el análisis del papel de las acciones impulsadas por el Gobierno de Cataluña desde los años 80 hasta la actualidad teniendo como referente las tendencias y líneas de futuro que se plantean a nivel internacional. Numerosas iniciativas y programas se han ido sucediendo a lo largo de los años en Cataluña sin que, en muchos casos, haya habido una continuidad y seguimiento de los resultados obtenidos. No obstante, las nuevas iniciativas sobre educación digital en Cataluña abren una esperanza ya que no están centradas exclusivamente en el uso de las tecnologías digitales, sino que la prioridad es la mejora del sistema educativo.
\end{abstract}

\section{Palabras clave}

Tecnologías digitales; Politicas educativas; Tendencias educativas 


\title{
Four decades of policies to integrate digital technologies in the classroom in Catalonia: actions, achievements and failures
}

\author{
Begoña Gros \\ bgros@ub.edu \\ Universitat de Barcelona \\ Joan-Anton Sánchez Valero \\ joananton.sanchez@ub.edu \\ Universitat de Barcelona \\ Iolanda García \\ igarciago@uoc.edu \\ Universitat Oberta de Catalunya \\ Cristina Alonso Cano \\ cristina.alonso@ub.edu \\ Universitat de Barcelona
}

\begin{abstract}
In most of the European countries, the implementation of digital technologies has been driven through government education policies. In this contribution, we will focus on the analysis of the role of actions promoted by the Government of Catalonia from the $80 \mathrm{~s}$ to the present taking into account the international studies about digital education. Numerous initiatives and programs have been supported in Catalonia over the years without, in many cases, a lack of continuity and evaluation of the results obtained. However, the new initiatives on digital education in Catalonia open new expectations. For the first time, the focus is not only the integration of digital technology, but also the improvement of education and not focused exclusively on the use of digital technologies.
\end{abstract}

\section{Keywords}

Digital technologies; Education policies; Educational trends 


\section{Tecnologías e innovación: una supuesta relación}

Desde sus inicios, la incorporación de las tecnologías digitales en la escuela ha estado asociada a la innovación educativa. El hecho de enseñar y aprender con tecnologías ha sido percibido como algo innovador en sí mismo, aunque, en muchas ocasiones, no se haya explicitado de forma clara el significado del término ni haya habido evidencias que lo avalen (Cuban, Kirkpatrick, \& Peck, 2001; Balanskat, Bannister, Hertz et al., 2013). Por el contrario, son numerosos los informes (por ejemplo: European Schoolnet, 2006; Valiente, 2010; Ranguelov, Horvath, Dalferth \& Noorani, 2011) que muestran cómo la introducción de las tecnologías digitales no necesariamente ha estado acompañada por cambios en las metodologías de enseñanza y aprendizaje.

Las investigaciones de los años 80 y 90 ofrecen escasos resultados sobre la incidencia de las tecnologías sobre los cambios metodológicos. En esos años, la mayoría de los estudios se centraron en ofrecer resultados globales basados en indicadores sobre el equipamiento tecnológico y la frecuencia de uso de los ordenadores (Carstens \& Pelgrum, 2006). De hecho, como señalan Sola \& Murillo (2011), el uso efectivo de la tecnología era muy limitado y no formaba parte de las actividades de aprendizaje diarias de los escolares por lo que era difícil que tuvieran una incidencia sobre los cambios en los métodos de enseñanza-aprendizaje.

El primer informe internacional que incorporó un análisis de la relación entre el uso de las TIC y la transformación metodológica es el SITES 2006 (Carstens \& Pelgrum, 2006), un estudio en el que participaron 28 países, incluido el programa de informatización de Catalunya. En el estudio se analizaron prácticas pedagógicas innovadoras que involucraban la utilización de tecnología. Los resultados muestran diferencias importantes entre los métodos de enseñanza. En general, el empleo de los métodos tradicionales no se ve afectado por las tecnologías digitales, ni en las competencias de los estudiantes ni en los resultados académicos. Sin embargo, aparecen correlaciones positivas entre los métodos innovadores y unas prácticas competenciales superiores en el manejo de las tecnologías por parte del alumnado.

Los estudios sobre la incidencia de la tecnología en las metodologías de enseñanza y aprendizaje se incrementan en el momento en que se empiezan a implementar los ordenadores portátiles en las aulas. Zheng, Warschauer, Lin \& Chang (2016), realizan un metaanálisis a partir de 65 artículos y 31 tesis doctorales entre el año 2001 y 2015, para examinar la incidencia de los programas 1 a 1 en el aprendizaje. En el análisis se concluye que el impacto de la utilización de los portátiles en el aula generalmente es positivo en todas las áreas disciplinares, especialmente en el aprendizaje de las matemáticas. No obstante, el efecto más importante no está en los contenidos del currículo sino en los tipos de aprendizajes. Se evidencia que los dispositivos portátiles se están utilizando ampliamente en cada etapa del proceso de escritura, desde la recopilación de la información, la planificación, la redacción, la edición, la recepción de comentarios entre iguales y la publicación. También se detecta una mayor autonomía en la obtención de recursos para el aprendizaje y un incremento de la comunicación entre los maestros, los alumnos y las familias. En definitiva, tal y como apuntan Gilleran (2006) y Pedró (2011), las tecnologías digitales no necesariamente han sido el desencadenante de la transformación del modelo de aprendizaje escolar, aunque la mayoría de las innovaciones educativas actuales utilizan las tecnologías digitales.

La implementación de las tecnologías digitales ha estado generalmente impulsada a través de políticas gubernamentales (Poggi, 2008; Mendonça \& Kaschny, 2019). En esta contribución, nos centraremos en el papel de las acciones impulsadas por el Gobierno de Cataluña desde los años 80 hasta los planes actuales y las propuestas futuras. En primer lugar, describimos las diferentes políticas promovidas desde el Gobierno centradas en el uso de la tecnología en la educación obligatoria. En segundo lugar, presentamos los planes de formación inicial y permanente del profesorado en tecnología educativa a lo largo de las tres últimas décadas. En el tercer apartado, se plantean las tendencias y líneas de futuro a nivel internacional, teniendo en cuenta los 
resultados obtenidos en materia de educación digital y, finalmente, presentamos las conclusiones sobre los aspectos aprendidos en el caso de Cataluña y que deberían guiar nuevas acciones de futuro.

\section{Las políticas educativas sobre tecnologías educativas en Cataluña}

Iniciamos este apartado presentando una síntesis (Tabla 1) de la evolución de las políticas educativas para impulsar el uso de las tecnologías digitales en Cataluña en los últimos 40 años.

\begin{tabular}{|c|c|c|}
\hline Período/Denominación & $\begin{array}{l}\text { Período/Denominación } \\
\text { Caracterización del período }\end{array}$ & Iniciativas más relevantes \\
\hline $\begin{array}{l}\text { 1982-1986 } \\
\text { Centre de Recursos d' } \\
\text { Informàtica Educativa i } \\
\text { Professional (CRIEP) }\end{array}$ & $\begin{array}{l}\text { - Inicialmente vinculado con la } \\
\text { Formación Profesional, que después } \\
\text { de expande a EGB y Bachillerato. }\end{array}$ & $\begin{array}{l}\text { - Proyecto EIX } \\
\text { - Proyecto TOAM } \\
\text { - Plan LOGO } \\
\text { - Centros CERO }\end{array}$ \\
\hline $\begin{array}{l}1986-2000 \\
\text { Programa d'Informàtica } \\
\text { Educativa (PIE) }\end{array}$ & $\begin{array}{l}\text { - Dotación de ordenadores y } \\
\text { software a los centros. } \\
\text { - Creación de software educativo } \\
\text { propio. }\end{array}$ & $\begin{array}{l}\text { - Convenio Bull (Secundaria). } \\
\text { - Red y portal XTEC. } \\
\text { - Base de datos SINERA. } \\
\text { - Proyecto de introducción } \\
\text { curricular de los medios } \\
\text { informáticos (EGB). } \\
\text { - Proyecto CAITI (FP). } \\
\text { - Proyecto ARGO. }\end{array}$ \\
\hline $\begin{array}{l}2000-2005 \\
\text { Subdirecció General de } \\
\text { Tecnologies de la } \\
\text { Informació (SGTI) }\end{array}$ & $\begin{array}{l}\text { - Integración en la estructura } \\
\text { orgánica del Departamento de } \\
\text { Enseñanza. } \\
\text { - Unificación de la informática } \\
\text { educativa y los medios } \\
\text { audiovisuales. }\end{array}$ & $\begin{array}{l}\text { - Portal Edu365. } \\
\text { - Conectividad generalizada } \\
\text { de banda ancha. } \\
\text { - Dotaciones en el marco de } \\
\text { proyectos estatales. } \\
\text { - Proyecto de integración } \\
\text { avanzada de les TIC (IATIC). }\end{array}$ \\
\hline $\begin{array}{l}\text { 2005-2008 } \\
\text { Àrea TIC }\end{array}$ & $\begin{array}{l}\text { - Área desvinculada del } \\
\text { Departamento de Educación }\end{array}$ & $\begin{array}{l}\text { - Proyecto Linkat. } \\
\text { - Proyecto Heura. }\end{array}$ \\
\hline $\begin{array}{l}2008-2019 \\
\text { Àrea de Tecnologies per a } \\
\text { I'Aprenentatge i el } \\
\text { Coneixement (Àrea TAC) }\end{array}$ & $\begin{array}{l}\text { - Voluntad de situar en igualdad de } \\
\text { condiciones los aspectos técnicos y } \\
\text { pedagógicos de las TIC que se ve } \\
\text { afectada por los cambios de } \\
\text { gobierno y la crisis económica. }\end{array}$ & $\begin{array}{l}\text { - Proyecto eduCAT1x1. } \\
\text { - Proyecto eduCat } 2.0 . \\
\text { - Iniciativa mSchools. } \\
\text { - Programa Tecnologías } \\
\text { Digitales para el Aprendizaje. }\end{array}$ \\
\hline $\begin{array}{l}2019 \\
\text { Àrea de Cultura Digital }\end{array}$ & $\begin{array}{l}\text { - Voluntad de situar las tecnologías } \\
\text { digitales en un marco global de } \\
\text { mejora educativa, incluyendo la } \\
\text { equidad. }\end{array}$ & $\begin{array}{l}\text { Proyecto mobils.edu. } \\
\text { - Proyecto de Educación } \\
\text { Digital 2020-2025. }\end{array}$ \\
\hline
\end{tabular}

Tabla 1. Cuatro décadas de políticas educativas en Cataluña. Adaptada y actualizada de Alonso, 2012, pág. 31.

A principios de los años 80 aparecen las primeras iniciativas del Departamento de Enseñanza de la Generalitat de Cataluña relacionadas con la informática educativa. Uno de los primeros proyectos fue la creación, en 1982, del Centro de Recursos de Informática Educativa y Profesional (CRIEP). Este centro, inicialmente vinculado con la formación de especialistas en el ámbito de la Formación Profesional (FP) en temas informáticos y en cuestiones pedagógicas y organizativas (Ruiz, 2007), 
después se expande a la Educación General Básica (EGB) mediante diferentes proyectos (TOAM, LOGO y los centros CERO) y en menor medida a los centros de secundaria (Proyecto EIX).

En 1986, el Departamento de Enseñanza crea el Programa de Informática Educativa (PIE) con una vigencia de cinco años que se va prorrogando hasta el año 2000. Durante el primer quinquenio, las actuaciones del PIE se centran en la dotación generalizada de equipamiento informático a todos los centros públicos de secundaria (Convenio Bull). A partir de 1990 las dotaciones se extienden a las escuelas de EGB (Proyecto de introducción curricular de los medios informáticos) mediante concursos de dotaciones vinculadas a proyectos de centro y a los centros de Formación Profesional (Proyecto CAITI). Paralelamente, en 1988 se pone en funcionamiento la Xarxa Telemàtica Educativa de Catalunya (XTEC), que cuenta con servicios de comunicación, noticiario, así como el acceso a bases de datos como el Sistema d'Informació Educativa i de Recursos per a I'Aprenentatge (SINERA), creada en 1987, que después de distribuirá en CD-ROM. Otro aspecto en el que se centra el PIE es en la creación de software educativo como el programa Clic. Con la aparición de Internet en 1995 la XTEC se convierte en un portal informativo, de experiencias y recursos educativos. El final del PIE coincide con las primeras fases del programa ARGO, que dota a todos los centros educativos de Cataluña de equipamientos informáticos y de software educativo.

En el año 2000, el Departamento de Enseñanza del Gobierno se reestructura y las políticas TIC son resituadas en un lugar preferente y relevante en la estructura del Departamento (Alonso, 2012) mediante la creación de la Subdirección General de Tecnologías de la Información (SGTI) adscrita a la Dirección General de Ordenación e Innovación Educativa, en la que se integran el Programa de Informática Educativa (PIE) y el Programa de Medios Audiovisuales (PMAV). Durante este período se sigue incidiendo en las dotaciones generalizadas a los centros, como la finalización del proyecto ARGO, y las realizadas en el marco de iniciativas estatales como el proyecto Internet en el Aula. También, a partir del 2002 se conectan todos los centros educativos a la banda ancha mediante fibra óptica. Con relación a los recursos en el 2001, se crea el portal educativo edu365 para familias y estudiantes, que incluye la versión en línea del programa Clic, ahora llamado JClic. En el año 2004 se pone en marcha el Proyecto de Integración avanzada de las TIC (IATIC), que dota de manera intensiva infraestructura informática a centros con una trayectoria consolidada en el uso de las tecnologías digitales.

En el año 2005 se crea el Área TIC, desvinculada del Departamento de Educación. Una iniciativa que parece querer impulsar la idea de las TIC como herramientas más que sus usos educativos (Alonso, 2012). Los proyectos de esta efímera etapa están centrados en dotaciones de infraestructuras de acceso a internet (Proyecto Heura), en la mejora del soporte técnico a los centros y en la promoción del software libre (Proyecto Linkat), que pese a ser una clara apuesta del nuevo gobierno de la Generalitat, no consigue los resultados esperados.

De nuevo, en el 2007 el Departamento de Educación modifica la política y, a diferencia de la anterior área TIC, se manifiesta una clara voluntad de situar en igualdad de condiciones los aspectos técnicos y pedagógicos. Para ello, se crea el Servicio de Tecnologías para el Aprendizaje y el Conocimiento (STAC), más tarde renombrado como Área TAC. En esta línea, en los diferentes portales educativos vinculados al STAC se visualizan algunos cambios asociados a la nueva concepción TAC: entornos más colaborativos, propuestas cooperativas, espacios de autoría y creación dirigidos a la comunidad educativa en un sentido amplio (Alonso, 2012). A pesar de ello, pronto se vislumbran incógnitas y contradicciones de esta nueva política. Por un lado, las dificultades de pasar de las TIC a las TAC (Sancho, 2008), por otro, la creación de un servicio en lugar de una subdirección general puede restar capacidad de decisión para abordar el desarrollo de esta nueva visión de las tecnologías digitales con el peligro que se quede en una mera declaración de buenas intenciones.

La principal contradicción de este nuevo discurso aparece en 2009 con la puesta en marcha del proyecto EduCAT1x1 en los primeros cursos de enseñanza secundaria, en el marco del proyecto 
estatal Escuela 2.0., y de los cambios en el liderazgo político del Departamento de Educación. Este proyecto vuelve a estar centrado en la mera introducción de ordenadores portátiles en el aula, desvinculándolo de la innovación y la mejora educativa. Las contradicciones con el nuevo discurso se agravan con la obligación de utilizar los ordenadores portátiles como soporte de libros de textos digitales y su carácter unipersonal, cuando en algunos docentes ya no trabajaban con este tipo material educativo (Alonso, Bosco, Corti y Rivera, 2014; Alonso, Guitert y Romeu, 2014).

En 2011, con un nuevo gobierno en la Generalitat, se impulsa el programa eduCat 2.0, que se focaliza de forma explícita en el impulso de la innovación metodológica. Se realiza una experiencia piloto, en unos centros seleccionados, que tiene como objetivo impulsar cambios metodológicos en los últimos cursos de primaria. Se utilizan portátiles de manera socializada, y es el centro quien decide los usos educativos a los que se destinan estos dispositivos. Se lleva a cabo un proceso de seguimiento y una evaluación externa de la experiencia (Gros, García y Durall, 2012) que pone de manifiesto que la mayoría del profesorado manifiesta que el uso de los portátiles y la conectividad promueven dinámicas más participativas y colaborativas que facilitan la adaptación de la clase a las diferentes necesidades, intereses y estilos de los alumnos. El profesorado también señala que cuando cada alumno tiene un dispositivo con conexión a Internet es absolutamente necesario cambiar la metodología, ya que no es posible limitarse a la mera transmisión de contenidos. Asimismo, inciden en que es necesario disponer de más tiempo para preparar los recursos y los materiales, valorando positivamente la creación de espacios de trabajo compartidos. Una vez finalizada la experiencia piloto estaba previsto poner al servicio de la comunidad educativa las actividades diseñadas por los profesores, en forma de propuestas de trabajo, pero esta difusión de las actividades no se llegó a realizar y el proyecto quedó en vía muerta.

Coincidiendo con la llegada de los recortes económicos en el sector educativo, aparece el programa mSchools, iniciativa de mEducation impulsada por Mobile World Capital Barcelona, en colaboración con la Generalitat de Cataluña, el Ayuntamiento de Barcelona y GSMA. Si bien esta iniciativa apuesta por promover nuevas formas de aprendizaje y contribuir a la mejora de la educación, es cuestionable el hecho de supeditar las políticas públicas educativas a iniciativas originadas en el sector tecnológico empresarial. En 2017 se inicia el programa de Tecnologías Digitales para el Aprendizaje, articulado en los siguientes ámbitos: la transformación digital del centro, las tecnologías móviles, la comunicación digital y la colaboración con entidades externas. Esta iniciativa, si bien supone una continuidad de la política iniciada en el año 2007, no está dotada económicamente.

En 2019 se produce una nueva y profunda reestructuración del Departamento de Educación, asociada al cambio político en la dirección de esta consejería. Se crea la Subdirección General de Innovación, Investigación y Cultura Digital con sus correspondientes subdirecciones generales y áreas vinculadas. Este incipiente concepto de Cultura Digital responde a la voluntad de situar las tecnologías digitales en un marco global de mejora educativa, incluyendo la equidad. Las primeras iniciativas de esta Subdirección General son el proyecto mobils.edu, con el objetivo de impulsar los dispositivos móviles, como herramienta educativa estratégica, y el ambicioso Plan de Educación Digital 2020-2025, que pretende asegurar la competencia digital de estudiantes y docentes, así como la creación de una red de centros digitalmente competentes en el marco de una transformación educativa del sistema educativo. 


\section{La formación en tecnologías educativas del profesorado no universitario en Cataluña}

\section{a. La formación inicial del profesorado en tecnologías digitales.}

Las tecnologías digitales aparecen reguladas por primera vez en las directrices de los planes de estudio del título de maestro de 1991 y contemplaban una materia troncal obligatoria para todas las especialidades del título "Nuevas tecnologías aplicadas a la educación. Recursos didácticos y nuevas tecnologías: utilización en sus distintas aplicaciones didácticas, organizativas y administrativas. Utilización de los principales instrumentos informáticos y audiovisuales".

El planteamiento instrumental de la asignatura, su estrechez de miras y su enfoque, fue ampliamente criticado por profesores universitarios, maestros y estudiantes (Sancho, Bosco, Alonso y Sánchez, 2015), a pesar de ello, algunos docentes que impartían la asignatura realizaron una apropiación de la misma, más allá de una formación técnico-instrumental basada en la selección, diseño y producción de materiales (Ornellas y Sánchez, 2008), posibilitando una reflexión conjunta con los estudiantes sobre lo usos de estas tecnologías.

En la legislación del año 2007 se regula la planificación de los actuales grados de maestro y se establecen las competencias que deben adquirirse relacionadas con las tecnologías digitales. En el grado de educación infantil: "conocer las implicaciones educativas de las tecnologías de la información y la comunicación y, en particular, de la televisión en la primera infancia". Y en el grado de educación primaria: "Conocer y aplicar en las aulas las tecnologías de la información y de la comunicación. Discernir selectivamente la información audiovisual que contribuya a los aprendizajes, a la formación cívica y a la riqueza cultural". A pesar de ello, estas competencias no se concretan en ningún módulo específico en tecnologías digitales, sino que se han de abordar en los diferentes módulos en que se estructuran los grados (Ornellas, Sánchez, Fraga y Domingo, 2015).

El paso de la asignatura obligatoria de la antigua diplomatura, a un planteamiento transversal en las diferentes asignaturas de los grados de educación infantil y educación primaria, no está dando los resultados esperados. Por un lado, se ha acentuado la preocupación respecto a que los estudiantes no reciban una adecuada formación en torno a las tecnologías digitales (Paredes, Guitert y Rubia, 2009; Herrada y Herrada, 2011; Losada, Valverde y Correa, 2012). Por otro, nos encontramos con que las tecnologías digitales tienen un tratamiento muy diferente en los planes de estudio de las diferentes universidades catalanas y que la opción por la transversalidad ha hecho que el profesorado que imparte las diferentes asignaturas dé un tratamiento desigual a las tecnologías digitales (Sánchez, Alonso, Camacho y Estebanell, 2018).

El Proyecto Interdepartamental de Competencia Digital Docente (PICDD) de la Generalidad de Cataluña ha sido un hito importante por el hecho de haber sido implementado de manera consensuada por todas las universidades catalanas, pero sobre todo por focalizarse en las habilidades de carácter didáctico y metodológico.

En la resolución de 2016 la Competencia Digital Docente (CDD) se define como "la capacidad que tienen los docentes de aplicar y transferir todos sus conocimientos, estrategias, habilidades y actitudes sobre las Tecnologías para el Aprendizaje y el Conocimiento (TAC) en situaciones reales y concretas de su praxis profesional con el fin de: (a) facilitar el aprendizaje de los alumnos y la adquisición de la competencia digital de este colectivo; (b) llevar a cabo procesos de mejora e innovación en la enseñanza de acuerdo con las necesidades de la era digital; y (c) contribuir a su desarrollo profesional de acuerdo con los procesos de cambio que tienen lugar en la sociedad y en los centros educativos."

Con el objetivo de analizar la presencia de la CDD en los grados de maestro de las universidades catalanas y hacer una propuesta de modificación de los planes de estudio de dichos grados para 
incorporar la CDD, profesores de todas las universidades participantes en el PICDD Ilevaron a cabo el proyecto Proposta interuniversitària de formació inicial de mestres en tecnologies Digitals (2015 ARMIF 00027), que fue seleccionada en la convocatoria de ayudas de investigación para la mejora de la formación inicial de maestros de la Generalitat de Catalunya. Los resultados del proyecto ponen de manifiesto: a) que la CDD tiene un tratamiento desigual en los planes de estudio de las diferentes universidades, y b) que en los estudios de grado hay carencias con relación a la CDD. A modo de ejemplo señalar, que no hay ningún descriptor en los grados de maestro de todas las universidades catalanas que contemple las dimensiones: Organización y gestión de espacios y recursos digitales; y Comunicación y colaboración (Domingo y Sánchez, 2018).

Tras recoger los posicionamientos de maestros y estudiantes en torno a la CDD se diseñó una propuesta de formación inicial de maestros en tecnologías digitales (Propuesta FIMTED) centrada en tres acciones: a) un tratamiento específico, mediante la incorporación de un módulo obligatorio de CDD; b) tratamiento integrado, que complementariamente al tratamiento específico que propone que los diferentes módulos de los planes de estudios integren los descriptores de la CDD y c) tratamiento profundizado, que propone que todas las universidades ofrezcan una mención en tecnologías digitales, o alternativamente, una o varias asignaturas optativas, articuladas a partir de un tratamiento profundizado de los descriptores de la CDD.

La Propuesta FIMTED fue asumida por la Conferencia de decanos de educación de Cataluña en la sesión de 27 de abril de 2018 y se acordó que cada facultad la desarrollaría teniendo en cuenta su contexto y características específicas. A pesar de ello, esta propuesta no es suficiente para asegurar que los estudiantes de los grados de maestro alcancen la CDD al finalizar su formación inicial, son necesarias más acciones para desarrollarla y concretarla metodológicamente, así como establecer mecanismos para su seguimiento y evaluación.

Con relación a la formación inicial del profesorado de Educación Secundaria, la formación pedagógica en el ámbito de las tecnologías digitales no estaba presente en el antiguo Certificado de Aptitud Pedagógica (CAP) y no lo está en el actual Máster de Formación del Profesorado de Secundaria (Ornellas, Sánchez, Fraga y Domingo, 2015). Uno de los requisitos para la verificación del máster de Profesor de Educación Secundaria, establece, entre las competencias que los estudiantes deben adquirir: "Buscar, obtener, procesar y comunicar información (oral, impresa, audiovisual, digital o multimedia), transformarla en conocimiento y aplicarla en los procesos de enseñanza y aprendizaje en las materias propias de la especialización cursada". Como en los grados de maestro, esta competencia no aparece de manera explícita en ningún módulo del máster.

\section{b. La formación permanente del profesorado en tecnologías digitales.}

En la evolución histórica de la formación permanente en tecnologías digitales en Cataluña podemos distinguir cuatro etapas (Tabla 2) que se corresponden con los diferentes planes de formación del profesorado del Departamento de Educación.

\begin{tabular}{|l|l|l|}
\hline Periodo & Caracterización del período & Conceptos clave \\
\hline $\begin{array}{l}1988- \\
1996\end{array}$ & $\begin{array}{l}\text { Formación como proceso de cualificación técnica que permita } \\
\text { generalizar el uso de las tecnologías digitales. }\end{array}$ & Introducción \\
\hline $\begin{array}{l}1996- \\
2001\end{array}$ & $\begin{array}{l}\text { Periodo de transición de los usos reproductores de los medios } \\
\text { a las tecnologías digitales como elemento de cambio e } \\
\text { innovación. }\end{array}$ & \\
\hline $2005-$ & Formación para contribuir a los procesos de cambio e & Impregnación \\
\hline
\end{tabular}




\begin{tabular}{|l|l|l|}
\hline 2010 & innovación mediante las TAC. & \\
\hline $2010-$ & Aparición de nuevas modalidades formativas. & Crisis económica \\
\hline
\end{tabular}

Tabla 2. Planes de Formación Permanente del Profesorado en Cataluña.

Con el Plan de Formación Permanente del Profesorado 1989-1996, que coincide con la creación del Programa de Informática Educativa, se inician acciones generalizadas de formación en el ámbito de las tecnologías digitales impulsadas por la Administración educativa de Cataluña. La formación, en este período, se concibe como un proceso de cualificación técnica del profesorado necesario para facilitar la generalización del uso de las tecnologías digitales en los centros educativos. Desde esta perspectiva se presupone que con el conocimiento instrumental de las herramientas tecnológicas se producirá, de manera automática, un uso pedagógico de estos recursos por parte de los docentes. Así, predomina una visión técnica sobre la enseñanza y la función del profesorado centrada en modelos de formación de entrenamiento que pretenden formar a los docentes como usuarios eficaces de las tecnologías digitales (Sancho, Ornellas, Sánchez, Alonso y Bosco, 2008).

El análisis de los objetivos del Plan de Formación Permanente del Profesorado 1996-2001, que coincide con la aparición de Internet, denota una forma de entender la integración curricular de las tecnologías digitales como elemento de cambio e innovación educativa que se acerca a una perspectiva tecnológica sobre y para la innovación educativa. Pese al énfasis "constructivista" de la reforma, la integración de las TIC en la formación del profesorado sigue basándose en los usos reproductores de los medios, es decir "aprender de la tecnología" (Ornellas, Sánchez, Fraga y Domingo, 2015).

El Plan de Formación Permanente del Profesorado 2005-2010 coincide con la creación del servicio de Tecnologías para el Aprendizaje y el Conocimiento (TAC) y marca un punto de inflexión en lo que se refiere al protagonismo dado a las tecnologías digitales en los procesos de enseñanza y de aprendizaje, poniendo el énfasis en las posibilidades educativas que ofrecen estas tecnologías contextualizadas en distintas formas de entender el currículum y en diferentes modelos pedagógicos. Asimismo, se dan orientaciones para una formación del profesorado en tecnologías digitales desde un modelo de desarrollo y mejora que contribuya a los procesos de cambio e innovación pedagógica a través de nuevas metodologías centradas en el alumnado, en la resolución de problemas, en el aprendizaje colaborativo y en la atención a la diversidad (Ornellas, Sánchez, Fraga y Domingo, 2015).

El cuarto periodo, desde el 2010 hasta la actualidad, se inicia con la irrupción de la crisis económica y los recortes en educación, que llevan al Departamento de Educación a encargar la impartición de los cursos de formación a personal técnico de dicho departamento y al personal de los Centros de Recursos Pedagógicos (CRP), dentro de su horario laboral. Las reticencias que genera esta situación llevan al Departamento de Educación a suprimir prácticamente todos los cursos de formación permanente, lo que comporta un crecimiento de la oferta privada de formación permanente, sobre todo vinculada a la acreditación de la Competencia Digital Docente. Frente a esta situación, el Departamento empezó a trabajar en nuevas modalidades formativas como la formación interna de centro y a la reconversión de las asesorías a centros por mentorías a profesores. También se produce un incremento de las jornadas formativas, concebidas como espacios de encuentro tematizados y de reconocimiento de experiencias realizadas. A partir de 2017, coincidiendo con el final de la crisis económica, se permite volver a remunerar la formación, se empiezan a recuperar los seminarios a los coordinadores TAC de los centros y los cursos en línea. 


\section{Propuestas de futuro y criterios para la elaboración de políticas de educación digital}

Podría decirse, añadiendo los matices y particularidades correspondientes, que las políticas para la integración de las tecnologías digitales en las escuelas no han tenido los resultados esperados ni han cubierto las expectativas en lo que respecta a la mejora de los procesos de enseñanza y aprendizaje, en ningún país del mundo. Los análisis y reflexiones realizados desde organismos internacionales, como la UNESCO, la OCDE, la Comisión Europea o la investigación desarrollada en diversos países, coinciden en esta conclusión y también en buena parte de los factores del diagnóstico. Un estudio desarrollado por expertos de cinco países, -China, Alemania, Japón, Italia y Estados Unidos- (Mao et al., 2019) concluye que la adopción de las tecnologías y la innovación en el ámbito educativo se ve obstaculizada por desafíos locales y sistémicos, que se encuentran anclados en las realidades económicas, culturales y sociales de cada contexto. En este sentido, los autores enfatizan la necesidad de investigar los factores contextuales que inciden en el diseño de las estrategias para la apropiación de la educación digital, entre ellos las características y limitaciones de los sistemas políticos, sociales y culturales que afectan al uso cotidiano de las tecnologías y a las prácticas educativas.

Butler et al. (2018) señalan, en este sentido, la importancia de lograr una alineación entre las políticas y los posicionamientos en materia de educación digital y el resto de los componentes del sistema educativo, así como aquellos que conectan las políticas educativas con el desarrollo económico y social de cada territorio. Mantienen que para posibilitar la transformación pedagógica, se debe garantizar una interrelación, o conectividad permeable (permeable connectivity) a la que se refiere Fullan (2006), en los tres niveles del sistema: a) nivel macro: factores contextuales o institucionales como normas culturales, dinámicas sociales, políticas educativas, o normativas curriculares; b) nivel meso: factores que tienen que ver con el ámbito escolar, como la infraestructura de Tecnologías Digitales disponibles, los planes de integración de las TIC, el liderazgo escolar, la trayectoria en innovación educativa y el perfil de las familias; c) nivel micro: factores individuales, referidos a los docentes (las prácticas pedagógicas, la organización del aprendizaje, la formación académica, la experiencia con el uso de las tecnologías, la actitud y creencias sobre el aprendizaje) y a los alumnos (perfil académico, social y cultural, necesidades educativas o experiencia con el uso de tecnologías digitales). Dicho de otra forma, de acuerdo con los autores, es necesario que las políticas de educación digital generen estrategias para promover la interacción, la sinergia y la influencia mutua dentro y entre estos tres niveles. Esto implica también que las medidas y prioridades a nivel nacional deben alinearse con los contextos y valores de las comunidades educativas locales, e involucrar y comprometer a los grupos y figuras de influencia en la toma de decisiones de las mismas. Para ello proponen un instrumento, el UNESCO framework, o "marco para la transformación de la educación por parte de las políticas de TIC", como marco de planificación estratégica y de análisis. Este marco comprende seis aspectos que podrían considerarse clave en un sistema de aprendizaje: visión de la tecnología en la educación, currículum y evaluación, pedagogía, práctica y desarrollo profesional docente, y organización y administración escolar. De esta forma, se propone abordar las implicaciones que los diferentes objetivos y visiones de las TIC pueden tener para los otros cinco componentes del sistema. Además, propone tres dimensiones o enfoques progresivos y complementarios, que conectan la política educativa con el desarrollo económico y social: alfabetización tecnológica, profundización del conocimiento y creación de conocimiento (Butler et al., 2018, p.477).

Siguiendo esta misma lógica, Ilomäki y Lakkala (2018), proponen el modelo Innovative Digital School model en Finlandia, como marco conceptual para la reflexión colectiva, la discusión y la planificación estratégica dentro de los centros escolares. El modelo integra los elementos que, de acuerdo con las autoras, son relevantes para la transformación de la escuela a través de las tecnologías digitales (Figura 1). A pesar de que actúa fundamentalmente en los niveles meso y 
micro y no en el macro, sí plantea que las políticas educativas deberían incorporar todos los componentes y partir de la creación de una visión compartida sobre el modelo de escuela digital que se pretende alcanzar y el tipo de desarrollo pedagógico que requiere.

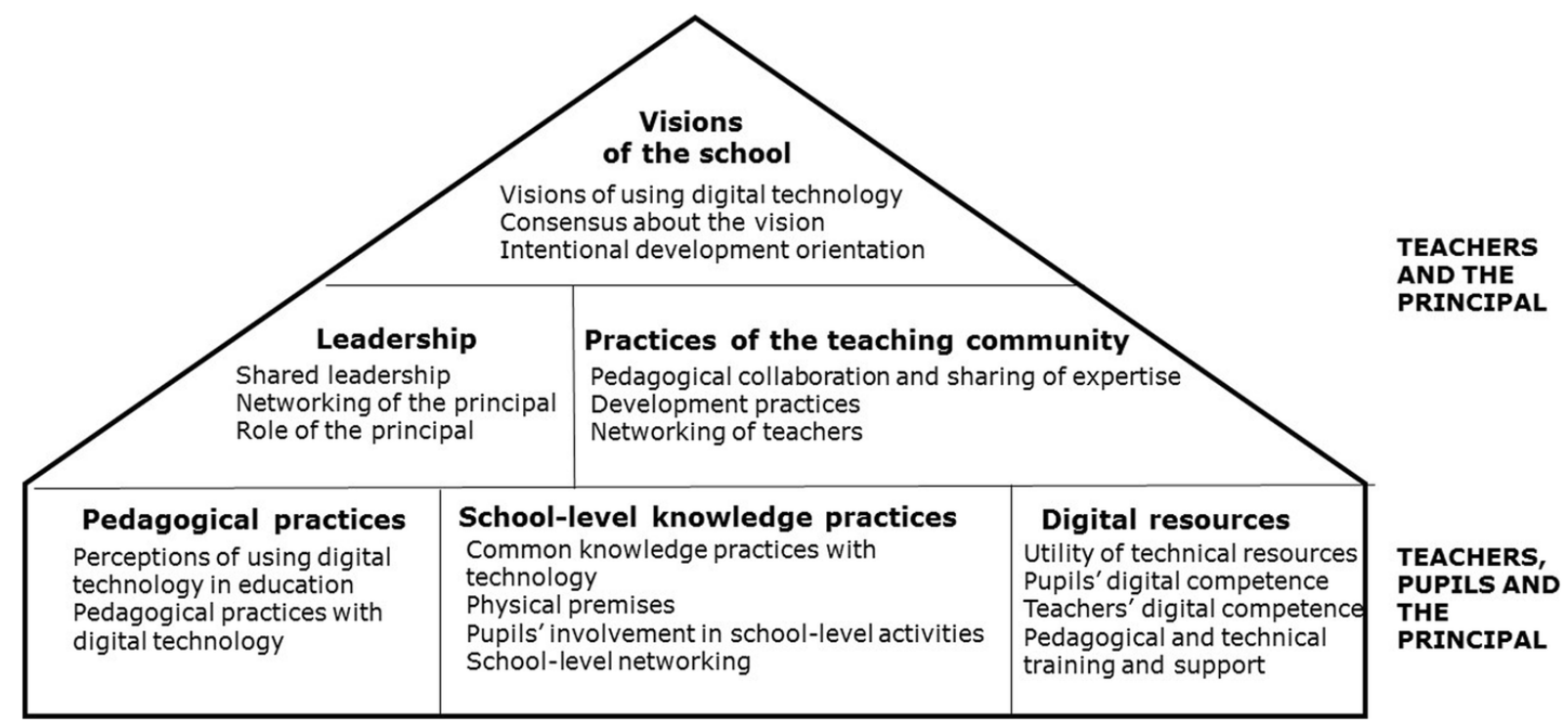

Figura 1: Innovative Digital School model (Ilomäki y Lakkala, 2018, p.9)

Si atendemos a las tendencias en lo que respecta al tipo de medidas específicas que se están proponiendo en este momento, encontramos el Plan de acción para la educación digital elaborado por la Comisión Europea (2018). El plan se orienta hacia la promoción, apoyo e incremento de las prácticas educativas digitales innovadoras. Incluye a todos los agentes involucrados desde una perspectiva de aprendizaje a lo largo de la vida, es decir, al sistema escolar pero también a las empresas, a los centros de investigación y a las ONG. Propone un total de 11 acciones distribuidas en las tres prioridades que se presentan a continuación:

1) Hacer un mejor uso de la tecnología digital para la enseñanza y el aprendizaje.

Dentro del primer punto se hace referencia al empoderamiento de los docentes y los educadores, a través del intercambio y la colaboración dentro del colectivo. Por otra parte, se destaca también la importancia de adoptar un enfoque institucional, poniendo a disposición de la comunidad educativa modelos y herramientas de seguimiento y evaluación (por ejemplo, la herramienta de autoevaluación SELFIE), que faciliten la adaptación al cambio desde una perspectiva sistémica: contemplando la capacitación en relación con las infraestructuras, los equipos, el currículum, los materiales y los modelos educativos. En este sentido, se destaca como punto clave el liderazgo en los centros educativos. La movilidad de docentes y estudiantes a través de intercambios se plantea también como una estrategia clave para complementar el trabajo desarrollado en el marco del proyecto de educación digital.

2) Desarrollar las competencias y capacidades digitales pertinentes para la transformación digital. 
El segundo punto plantea un enfoque a lo largo y a lo ancho de la vida para el desarrollo de la competencia digital, según el cual, no solo debe estar integrado en el currículum escolar, sino aprovechar otros espacios de educación no formal. Además, se insiste en que debe incidir en el desarrollo de capacidades para crear las tecnologías y no solo para utilizarlas; la preparación para el desarrollo de capacidades digitales avanzadas que permitan, por ejemplo, el uso de datos abiertos y herramientas colaborativas digitales, se considera necesario para formar a futuros investigadores e innovadores. Otro aspecto fundamental es reforzar entre los alumnos, pero también entre los docentes y las familias, el pensamiento crítico y la alfabetización mediática, para hacer frente de manera efectiva a cuestiones relativas a la seguridad en línea, la higiene cibernética y la manipulación informativa. La superación de la brecha de género de la educación y el emprendimiento digital es otra de las metas. Para ello se propone ofrecer modelos de referencia y apoyo, que ayuden a superar los estereotipos de género en el campo de las TIC.

3) Modernizar la educación mediante un mejor análisis de los datos, que permita la predicción y garantice la igualdad y la calidad del acceso a las infraestructuras digitales.

Finalmente, la tercera prioridad pone el énfasis en la mejora del análisis y la comparativa de los datos a gran escala, para ampliar y profundizar en el conocimiento sobre las distintas iniciativas de educación digital en Europa. Entre otras acciones, la Comisión se propone desarrollar, conjuntamente con la OCDE, un nuevo módulo PISA sobre el uso de las tecnologías en la educación. Se propone también ampliar los programas prácticos para investigar y experimentar en el campo de las analíticas de aprendizaje y la inteligencia de datos, con el fin de desarrollar instrumentos y orientaciones que contribuyan a abordar problemas concretos y a mejorar la aplicación y el control de las políticas educativas digitales. Por último, valora como necesario el grado de implicación de los propios destinatarios, docentes, alumnos y familias, en el impulso de la innovación con la finalidad última de facilitar la adopción de soluciones innovadoras.

Por otra parte, la Comisión Europea ha publicado recientemente el informe Digital education policies in Europe and beyond: key design principles for more effective policies (Conrads et al. 2017), en el que proporciona pautas para mejorar el diseño de futuras políticas en el ámbito de la educación y las tecnologías. Con esta finalidad realiza un análisis de más de cuarenta iniciativas políticas europeas de nivel nacional y regional, seis de ellas en profundidad. El informe revela una tendencia general hacia una mayor integración de la educación digital en las políticas generales de educación e innovación, sin que ello signifique perder concreción a nivel operativo. El propósito es abordar los puntos débiles más generalizados en este tipo de políticas al mejorar la coherencia de la estructura general, facilitar la disponibilidad de recursos y garantizar la sostenibilidad en el tiempo. La estrategia propuesta para el despliegue de las medidas en este ámbito sigue un esquema cíclico de menor a mayor envergadura. Se inicia con exploraciones e indagaciones locales que van generalizándose a medida que se va consolidando y confirmando su efectividad. Se da mucha importancia al hecho de que las medidas políticas estén bien ancladas en el contexto específico de implementación, por lo que se sugiere empezar con un análisis en profundidad de cada contexto y de sus necesidades específicas. Para ello, se plantea como indispensable disponer e implementar sistemas de seguimiento y evaluación que permitan valorar e ir ajustando las iniciativas a lo largo de su desarrollo. Se considera que tanto las estrategias bottom-up como topdown pueden funcionar y se aboga por una combinación de ambas. En cuanto al contenido o líneas estratégicas más destacadas, se plantean a un mismo nivel la mejora de las infraestructuras y la formación docente, además del desarrollo y/o uso de recursos digitales. 
A continuación, nos referimos a los ocho principios básicos para el diseño de políticas educativas digitales propuestos en el informe de Conrads et al. (2017). Complementamos algunos de los puntos con los resultados y reflexiones procedentes de otros estudios e informes. Consideramos que estos principios proporcionan una hoja de ruta básica para la elaboración y el despliegue de políticas y medidas concretas en materia de educación digital:

a) Utilizar un enfoque holístico dirigido al cambio sistémico:

La implementación de iniciativas que abarcan distintas dimensiones y niveles tienen el potencial de ser disruptivas con el status quo, cosa que favorece una transformación en profundidad. Esto se debería combinar con objetivos suficientemente claros y concretos para poder llevar a cabo valoraciones y extraer conclusiones. Adoptar un enfoque holístico implica actuar en los niveles macro, meso y micro, antes mencionados, y considerarlos a su vez como vasos comunicantes. El enfoque holístico favorece a su vez una mirada sostenible a nivel local, que prioriza la creación de capacidad y los recursos y aplicaciones locales, así como el uso de tecnologías innovadoras de bajo coste.

b) Establecer una visión a largo plazo con objetivos viables a corto plazo:

Las intervenciones deberían ser evaluables a largo plazo y no depender de ciclos políticos cortos o cambios en las prioridades de los gobiernos. La financiación y el apoyo también deberían estar garantizados a largo plazo. Esta visión a largo plazo debería, no obstante, convivir con la formulación de objetivos a corto plazo que puedieran integrarse en un marco de evaluación de la política desde el comienzo de su puesta en marcha. Esto permitiría obtener resultados concretos de forma periódica, que podrían difundirse entre el público general y también entre los responsables de la toma de decisiones.

c) Implementar la tecnología como un medio, no como un fin:

El uso de la tecnología debería quedar integrada en el conjunto de cuestiones relativas a la educación y no tratarse como un aspecto independiente, especialmente en lo que respecta a las prácticas pedagógicas y a la formación del profesorado. El caso de Australia puede resultar paradigmático en este sentido. El currículum escolar contempla la promoción del uso de las tecnologías digitales a través de los proyectos STEM (Science, Technology, Engineering and Mathematics) (Masters et al., 2018). De esta forma, se han previsto fondos adicionales en esta área para que las escuelas inviertan en equipamiento y desarrollo profesional para desarrollar adecuadamente el nuevo currículum en tecnologías en dos áreas: a) diseño y tecnologías, donde los estudiantes utilizan el pensamiento y las tecnologías de diseño para generar y producir soluciones a necesidades y oportunidades auténticas; y b) tecnologías digitales, donde los estudiantes utilizan el pensamiento computacional y los sistemas de información para definir, diseñar e implementar soluciones digitales. Se trata, por lo tanto, de abordar la alfabetización digital desde una perspectiva global, construyendo el pensamiento computacional a través de proyectos que utilizan la tecnología para el desarrollo de la lógica, la creatividad y el trabajo en equipo. En definitiva, de las competencias básicas para enfrentarse a los retos de la sociedad actual. En el marco de este planteamiento se pone especial énfasis en el aprendizaje de la programación, así como en el uso de la inteligencia artificial, la realidad aumentada y la realidad virtual en los centros educativos (DET, 2017). Masters et al. (2018) señalan la necesidad de alinear la investigación con estos proyectos, con la finalidad de incorporar un componente de comprensión, reflexión y rigor, que permita apoyar decisiones informadas sobre la presencia de las tecnologías digitales en el currículo. Por su parte, la UNESCO (2017) advierte de la necesidad de aprovechar las innovaciones digitales para garantizar un aprendizaje basado en la equidad. Tecnologías emergentes como la inteligencia artificial, el 
big data y las analíticas de aprendizaje prometen grandes avances relacionados con la personalización del aprendizaje, y la optimización de los procedimientos de seguimiento y evaluación. Sin embargo, también plantean cuestiones críticas como el reemplazo de los docentes, la ética de los datos y la brecha digital.

d) Adoptar la experimentación, asumiendo el riesgo y también los fracasos:

Permitir un mayor grado de experimentación en procesos de ensayo y error flexibles e iterativos, que faciliten el ajuste de las políticas a lo largo de un periodo de tiempo, a partir de las evaluaciones realizadas en intervalos regulares. Apoyar la investigación que oriente el diseño de futuros modelos escolares y de aprendizaje, de manera alineada con las condiciones de cada contexto local (UNESCO, 2017).

e) Considerar la importancia y también los límites de la evaluación del impacto:

Los mecanismos de seguimiento y evaluación deberían estar estructurados en un marco temporal con retos viables definidos y espacios de explicitación y valoración establecidos a priori, que permitan reorientar o corregir aspectos concretos. Además, deberían incorporar las últimas tendencias en evaluación y someterse a una revisión permanente. A su vez, teniendo en cuenta la complejidad del objeto de evaluación, deberían establecerse criterios y aproximaciones suficientemente multidimensionales y no limitarse a los aspectos fácilmente cuantificables. Los sistemas de datos pueden informar la planificación, implementación y evaluación de las políticas. Por lo tanto, es necesario establecer pautas y políticas adecuadas sobre la recopilación, gestión y uso ético de las eviencias educativas (UNESCO, 2017).

f) Involucrar a todos los implicados en un diálogo estructurado:

Consultar y tener en cuenta a todas las partes interesadas puede ayudar a garantizar que las medidas planificadas se orienten hacia los aspectos prioritarios. Además, facilita la aceptación y la adopción de las medidas y promueve la participación por parte de los agentes implicados.

g) Escuchar lo que tengan que decir las escuelas y los docentes:

La motivación e implicación de los referentes en las escuelas, los directores de centros educativos y el colectivo docente en general es fundamental para impulsar políticas relacionadas con la innovación educativa. Para ello es importante garantizar un margen de libertad de acción, flexibilidad y autonomía, que favorezca la apropiación de las medidas implementadas en cada contexto y situación, cosa que a la vez permitirá obtener resultados a través de las mismas políticas, bajo condiciones distintas.

h) Desarrollar la Competencia Digital Docente (CDD):

La formación inicial del profesorado y el desarrollo profesional docente en la mejora de sus competencias digitales para el uso de las tecnologías digitales en la práctica educativa, son requisitos esenciales para empoderar a este colectivo y asegurar la implementación de las políticas de educación digital y lograr cambios tangibles en las prácticas de enseñanza y aprendizaje. En este sentido, la UNESCO (2017) propone el desarrollo de estándares de competencia docente, así como el apoyo a la consolidación de comunidades de práctica de profesores que actúen como catalizadores de buenas prácticas y desarrollo profesional. El movimiento de educación abierta, abanderado en la creación de comunidades y repositorios de REA y MOOC, podría continuar desempeñando un papel importante en este sentido, a pesar de que hay cuestiones difíciles de resolver que tienen que ver con la certificación, la acreditación, las licencias y el acceso a contenido de calidad. 


\section{Conclusiones: un largo camino de aprendizaje}

Tal y como hemos señalado previamente, es esencial que las políticas sobre tecnología educativa estén alineadas con las prioridades y medidas en materia de educación del conjunto del sistema educativo. En Cataluña, sin embargo, los sucesivos planes gubernamentales descritos no han tenido en consideración este aspecto y se han centrado en la dotación tecnológica de los centros y en aspectos parciales de la capacitación del sistema, sin un enfoque suficientemente global. Este aspecto se evidencia claramente en la formación del profesorado impulsada en los años ochenta y noventa, cuyas propuestas formativas no se integran en ningún plan de innovación o propuesta de cambio del sistema educativo, sino que se limitan a proporcionar conocimientos tecnológicos.

Un aspecto muy preocupante es que las diferentes políticas no han estado acompañadas de estudios sistemáticos de evaluación de los resultados, que hayan permitido extraer conclusiones concretas que pudieran mostrarse a la comunidad educativa y a los responsables políticos. La financiación y el apoyo ha dependido directamente de los diferentes gobiernos del momento. Quienes han ido modificando las prioridades sin un posicionamiento y perspectiva manifiesta, ni mucho menos una planificación, que garantizase la sostenibilidad de las medidas adoptadas a medio y largo plazo.

En la educación obligatoria, el uso de las tecnologías digitales se ha acompañado de un discurso sobre la innovación educativa más cosmético que real, puesto que no ha incidido en las bases de la práctica educativa para lograr una transformación real de las mismas. Por otra parte, tampoco se ha implicado a la comunidad educativa, empezando por el colectivo docente, como agente de cambio fundamental, en las medidas relacionadas con la innovación educativa. Así, las políticas sobre el uso de las tecnologías digitales en la escuela se han centrado de forma sistemática en los aspectos tecnológicos, y se han elaborado sin una participación e implicación de los referentes escolares y del colectivo docente en general.

Tal y como hemos evidenciado a lo largo del artículo, en la formación inicial y permanente del profesorado ha predominado un enfoque instrumental basado en modelos muy tecnocéntricos que no han tenido en cuenta la capacitación global del profesorado en los usos y apropiaciones de las tecnologías digitales para el aprendizaje en todas sus implicaciones.

Las directrices políticas referidas al desarrollo y utilización de recursos educativos digitales se han desarrollado a partir de criterios heterogéneos, dispares y en ocasiones contradictorios y más bien dependientes del origen más o menos fortuito de las inversiones del momento. Por este motivo, no ha habido claridad ni continuidad en las propuestas como se evidencia en la implementación de los programas mencionados: EduCAT 1x1, EduCat 2.0, mSchools, mEducation. Por otra parte, no ha habido incentivos o iniciativas relacionadas con el diseño y la creación de productos tecnológicos, así como oportunidades para pilotar y evaluar su implementación en las aulas.

En este sentido, la autonomía y las figuras de liderazgo de los centros educativos son elementos fundamentales para la construcción y el sostenimiento de un proyecto digital suficientemente sensible y responsable con cada contexto específico. El proyecto digital de cada centro educativo debería partir de los ejes de la propuesta general, pero a la vez adaptarse a su propio plan educativo, y de esta forma insertarse y recorrer sus líneas prioritarias de actuación de manera transversal.

Las iniciativas sobre educación digital en Cataluña, iniciadas a raíz de la reestructuración del Departamento de Educación en 2019, y en especial el Plan de Educación Digital 2020-2025, suponen un nuevo punto de inflexión, que no se focaliza en el uso de la tecnología en sí sino en la mejora de la educación. Es decir, las propuestas no están centradas exclusivamente en una visión artefactual de la tecnología, sino que ésta se concibe como herramienta para la transformación educativa, poniendo el énfasis en la mejora y la equidad como ejes clave. Este plan se inscribe de manera explícita dentro del marco de referencia de la Comisión Europea, en cuanto a sus 
programas para el desarrollo de la Competencia Digital (DigComp), tanto en la dimensión del alumnado, como del profesorado y la comunidad integrada por una red de centros educativos de todo el territorio. También se alinea con las directrices de la OCDE, la UNESCO y la ONU en sus objetivos de equidad y desarrollo sostenible. Pretende abarcar, por lo tanto, los tres niveles mencionados por Fullan (2006) macro, meso y micro, e incorpora también una propuesta de seguimiento y evaluación de las acciones implicadas.

Con todo, genera, como en la reestructuración llevada a cabo en 2007, incógnitas e inquietudes con relación a la eficacia de planes generalizados de implementación de mejoras, la financiación de dichos planes, así como la sostenibilidad dentro de un contexto de inestabilidad política y económica. Si atendemos a los criterios propuestos por Conrads et. al. (2018) parece estar orientado en la dirección correcta. El Plan se presenta bajo el leitmotiv de "capacitar a todos los alumnos para aprender en un mundo digital", lo cual implica incorporar la tecnología digital en el aprendizaje a lo largo y ancho de la vida, pero hacerlo además con el propósito de dar respuesta a los retos que la sociedad digital plantea. Esto supone superar el debate de la tecnología en el aula, para y pasar a contemplarla como palanca de transformación educativa. Otro de los grandes retos es que esto se consiga sin renunciar a los principios de inclusión y, por lo tanto, teniendo en cuenta a todo el alumnado, en toda su diversidad. Será necesario valorar las medidas concretas que se ponen en marcha y el apoyo específico que se brinda a los centros educativos y al profesorado, así como su capacidad para generar complicidades y sinergias con el conjunto del sistema educativo y con el tejido social de cada territorio.

\section{Referencias}

Alonso (2012). La evolución de las políticas de uso de las TIC en la educación en Cataluña. En J. M. Sáncho y C. Alonso (comps.) La fugacidad de las políticas, la inercia de las prácticas, pp. 115-138. Barcelona: Octaedro.

Alonso, Bosco, Corti i Rivera (2014). Prácticas de enseñanza mediadas por entornos 1X1: Un estudio de casos en la educación obligatoria en Cataluña. Profesorado: Revista de currículum y formación del profesorado, 18(3), 99-118.

Balanskat, A., Bannister, D., Hertz, B., Sigillò, E., \& Vuorikari, R. (2013). Overview and Analysis of 1:1 Learning Initiatives in Europe. JRC Scientific and Policy Report. Luxembourg: Publications Office of the European Union.

Butler, D., Leahy, M., Twining, P., Akoh, B., Chtouki, Y., Farshadnia, S., ... y Valtonen, T. (2018). Education Systems in the Digital Age: The Need for Alignment. Technology, Knowledge and Learning, 23(3), 473494. DOI: $10.1007 / \mathrm{s} 10758-018-9388-6$

Carstens, R. y Pelgrum, W. J. (2009). Second Information Technology in Education Study: SITES 2006 Technical Report. Amsterdam: International Association for the Evaluation of Educational Achievement.

Conrads, J., Rasmussen, M., Winters, N., Geniet, A. y Langer, L., (2017). Digital education policies in Europe and beyond: key design principles for more effective policies. Luxemburgo: Publications Office of the European. DOI: $10.2760 / 462941$.

Cuban, L., Kirkpatrick, H., \& Peck, C. (2001). High access and low use of technologies in high school classrooms: Explaining an apparent paradox. American educational research journal, 38(4), 813-834.

Department of Education and Training of Australia (2017). Support for science, technology, engineering and mathematics. Recuperado de: https://www.education.gov.au/support-science-technology-engineeringand-mathematics

Domingo, M. y Sánchez, J. A. (2018). Construyendo una propuesta sobre competencia digital docente para la formación inicial de maestros de Cataluña. En I. del Arco i P. Silva (eds.) Tendencias nacionales e internacionales en organización educativa: entre la estabilidad y el cambio, pp 1-7. Madrid: Wolters Kluwer.

European Commision (2018). Communication from the Commission to the European Parliament, the Council, the European Economic and Social Committee and the Committee of the Regions. On the Digital Education Action Plan. Bruselas. Recuperado de: https://ec.europa.eu/education/education-in-theeu/digital-education-action-plan_en 
European Schoolnet, (2006). The ICT Impact Report: A review of studies of ICT impact on schools in Europe. Bruselas: European Commission.

Fullan, M. (2006). Change theory: A force for school improvement. Seminar Series Paper No. 157. Centre for Strategic Education. Recuperado de: http://www.michaelfullan.ca/Articles_06/06_change_theory.pdf

Gros, B., García, I. y Durall, E. (2012). Experiència pilot EduCAT 2.0 a Educació Primària. Informe de evaluación. Recuperado de: http://xtec.gencat.cat/web/.content/alfresco/d/d/workspace/SpacesStore/0010/2f138996-6bad-4132acac-f82c5cb3cf50/informe_avaluacio_eduCAT2.0.pdf

Ilomäki, L. y Lakkala, M. (2018). Digital technology and practices for school improvement: innovative digital school model. Research and practice in technology enhanced learning, 13(1), 25. Recuperado de: https://telrp.springeropen.com/articles/10.1186/s41039-018-0094-8

Losada, D., Valverde, J. y Correa, J. M. (2012). La tecnología educativa en la universidad pública española. PixelBit: Revista de medios y educación, 41, 133-148.

Mao, J., Ifenthaler, D., Fujimoto, T., Garavaglia, A. y Rossi, P. G. (2019). National Policies and Educational Technology: a Synopsis of Trends and Perspectives from Five Countries. TechTrends, 63(3), 284-293. Recuperado de: https://link.springer.com/article/10.1007/s11528-019-00396-0

Masters, J. (2018). Trends in the digitalization of K-12 Schools: the australian perspective. In Seminar.net, 14(2) 120-131. Recuperado de: https://journals.hioa.no/index.php/seminar/article/view/2975

OECD (2017) The OECD Handbook for Innovative Learning Environments. Paris: OECD Publishing. DOI: https://dx.doi.org/10.1787/9789264277274-en

Ornellas, A. y Sánchez, J. A. (2008). Una mirada hacia la formación del profesorado en Tecnologías de la Información y la Comunicación en Cataluña. Diálogos. Educación y formación de personas adultas, 55(II), 49-62.

Ornellas, A., Sánchez, J. A., Fraga, L. y Domingo, L. (2015). Políticas y prácticas en la formación permanente del profesorado en TIC en Cataluña. Revista Electrónica Interuniversitaria de Formación del Profesorado, 18(3), 83-96. doi: 10.6018/reifop.18.3.190271

Paredes, J, Guitert M. y Rubia B. (2015). La innovación y la tecnología educativa como base de la formación inicial del profesorado para la renovación de la enseñanza. Revista Latinoamericana de Tecnología Educativa, 14(1), 101-114.

Pedró, F. (2011). Tecnología y escuela. Lo que funciona y por qué. Madrid: Fundación Santillana.

Poggi, M (Coord) (2008). Las TIC: del aula a la agenda política. UNESCO- UNICEF: Buenos Aires.

Ranguelov, S., Horvath, A., Dalferth, S. y Noorani, S. (2011). Key Data on Learning and Innovation through ICT at School in Europe 2011. Bruselas: Education, Audiovisual and Culture Executive Agency.

Ruiz, F. (2007). Vint-i-cinc anys de polítiques d'integració de les TIC als centres docents de Catalunya. En C. Sigalés, J. M. Momimó y J. Meneses (Coord.), L'escola ala societat xarxa: Internet a l'educació primària i secundària. IN3-UOC. Recuperado de: https://www.uoc.edu/in3/pic/cat/pdf/pic_escola_capitol2.pdf

Sánchez, J. A., Alonso, C., Camacho, M. y Estebanell, M. (2018). Análisis de la presencia de la Competencias Digitales Docentes en los planes de estudio de los grados de las universidades catalanas. En P. Membiela, N. Casado, M. I. Cebreiros y M. Vidal (eds.) Investigación y práctica en la educación superior, pp. 187-192. Orense: Educación Editora.

Sancho, J. M.; Ornellas, A.; Sánchez, J. A.; Alonso, C. y Bosco, A. (2008). La formación del profesorado en el uso educativo de las TIC: una aproximación desde la política educativa. Praxis Educativa, 12, 10-22.

Sancho, J. M., Bosco, A., Alonso, C. i Sánchez, J. A. (2015). Formación del profesorado en Tecnología Educativa: de cómo las realidades generan los mitos. Revista Latinoamericana de Tecnología Educativa, 14(1), 17-29. doi: 10.17398/1695-288X.14.1.17

Sola, M. \& Murillo, F. (2011). Las TIC en la Educación: Realidad y expectativas. Informe Fundación Telefónica. Madrid: Ariel.

UNESCO (2017) Leveraging information and communication technology to achieve Education 2030: report of the UNESCO 2017 International Forum on ICT and Education 2030. Qingdao, China. Recuperado de: https://unesdoc.unesco.org/ark:/48223/pf0000259587

Valiente, O. (2010): 1-1 in EduCATion: Current Practice, International Comparative Research Evidence and Policy Implications, OECD EduCATion Working Papers, No. 44. OECD Publishing.

Zheng, B., Warschauer, M., Lin, C. H., \& Chang, C. (2016). Learning in one-to-one laptop environments: A meta-analysis and research synthesis. Review of Educational Research, 86(4), 1052-1084. 